\begin{abstract}
A large number of publications recognize that there are melanocytic lesions with microscopic features similar to melanoma, related to their location, with no prognostic importance. Those locations are represented by the ear, the milk lines (axillary, breast, periumbilical and inguinal regions), palms, soles and flexural regions. The periumbilical nevi are included by some authors in the category of the flexural nevi. In 2004, Rongioletti et al., performed a study on a number of 101 breast nevi, considering 10 histologic parameters. Starting from Rongioletti's study we measured 10 histologic parameters on 121 nevi ( 26 from the periunmbilical area) and notes with 0 if absent or impossible to evaluate and with 1 if present. The score for each lesion ranged from 0 to 6 and we compared the features of the periumbilical nevi with the nevi from the control sites and found that the ones in the periumbilical area have more frequently atypical features than the nevi from the other sites (lemtiginous proliferation of nevus cells, architectural disorder of the nevus cell nests and stromal reactions as dermal fibroplasias and dermal lymphocytic infiltrate). We also performed immunohistochemical examination on lesions that presented three or more of
\end{abstract}

\section{Mariana Aşchie,}

Department of Pathology, Faculty of Medicine, "Ovidius” University, Constanta, Romania

Clinical Service of Pathology, Emergency County Hospital, 145 Tomis Avenue, 900591 Constanta, Romania

Phone +40241503289

aschiemariana@yahoo.com the examined histologic parameters, but the results were not very suggestive. The conclusion of this study is that the atypical features of the breast nevi are only site related atypias and have no hormonal influences.

Keywords: nevi, special sites, periumbilical, atypia.

\section{Introduction}

It is recognized in a large number of publications that there are some sites of melanocytic nevi that can present aspects similar to melanoma. Those sites include the head and neck (specially the ear), the "milk line" (axillary, breast, periumbilical and inguinal), acral regions (palms and soles) and flexural regions. The microscopic aspect of these nevi necessitates sometimes differentiation from melanoma [1,2].

The milk lines are the progenitors of mammary glands and nipples. All the mammals (both male and females) present a pair of symmetrical mammary ridges, along which the mammary gland the areolas and the nipples develop. Those ridges appear around six weeks of fetal life and they go from the axillary region, down the torso to the groin and sometimes to the inferior limbs. Around the 9th week most of 
the milk lines disappear, except those on the torso. Occasionally the milk lines persist and therefore some individuals may present ectopic mammary tissue or multiple nipples $[2,3]$.

In 2000, F. Rongioletti and a group of collaborators performed a study on 40 melanocytic nevi located on flexural sites (axilla, umbilicus, inguinal creases, pubis, scrotum and perianal area). The purpose of this study was to evaluate if these nevi share any of the atypical features of the genital nevi. They demonstrated that the nevi in the flexural sites present a more discohesive pattern, similar to the genital nevi. From the 40 studied cases, 20 $(55,5 \%)$ presented this feature, with confluence of the nevus cells nests, that have various sizes and shapes, and diminished cohesion of melanocytes [5]. In 2004 Rongioletii and a group of pathologists associated with Melanocytic Lesion Group of the Italian Association of Dermatopathoogy, performed an interinstitutional study of 101 breast nevi which proved that breast nevi do not have many histological differences from the flexural ones, but they have more atypical features such as intraepidermal melanocytes, melanocytic atypias and dermal fibroplasia.[6] The role of the estrogen in the biologic behavior and the histologic aspect is still unsure, because there were no major differences between males and females. Starting from this study we have analyzed the periumbilical nevi (as part of the milk line) in order to obtain a histopathologic and immunohistochemical model $[1,4,5,6]$.

\section{Materials and methods}

This study includes 121 melanocytic nevi localized on the torso and the abdomen, 26 of them from the periumbilical area. Those lesions have been diagnosed in the Pathology Department of Clinical Emergency County Hospital of Constanta, Romania during 2007-2011. They have been categorized by conventional criteria in junctional, dermal, mixed and dysplastic nevi. For every one of these nevi we measured the 10 parameters proposed by Rongioletti and colab, such as: 1) asymmetry, 2) absence of demarcation of melanocytes at lateral margins of the lesion, 3) lentiginous proliferation of melanocytes for three consecutive papillary ridges, 4) nested and discohesive pattern with bridging of nests between rete ridges, 5) intraepidermal melanocytes singly or in groups, above the basal layer over the length of three rete ridges, 6) involvement of the hair follicle by melanocyte proliferation, 7) absence of maturation of deep dermal melanocytes, 8) melanocyte atypia including one of the following: prominent nucleoli, irregular chromatin pattern, increase of nuclear/ cytoplasmic ratio, finely distributes melanin pigment within the cytoplasm (smoky cytoplasm), 9) fibroplasia of the papillary dermis and 10) lymphocytic infiltrate in the dermis. Each parameter was scored 1 if present and 0 if absent or impossible to evaluate. For every lesion we established a score with a minimum value of 0 and maximum of 10 . The score of the nevi from the periumbilical area was compared with the score from the nevi in the other sites of the torso and also the scores were compared between man and women as well as different age groups. For lesions with scores above 3 we used specific melanocytary immunohistochemical stains such as HMB 45, Melan A and S100, proliferation markers (Ki67) and $\alpha$-estrogen receptors markers. Immunohistochemical studies were performed on formalin-fixed, paraffin tissues sections using a Dako Envision TM + Dual Link System HRP (Dako, Carpinteria, CA, USA) according to manufacturer's instruction and counterstained with haemathoxylin. The results were statistically analyzed by student t-test and chi-square test using Microsoft Excel and MedCalc 12.

\section{Results}

We collected 121 nevi, 26 of them from the periumbilical area and 95 from the thorax (exclusive the breast area) and upper abdominal wall. Out of these 
nevi, 97 are from women (20 from the periumbilical area and 77 from the control sites) and 24 from men ( 6 from the periumbilical area and 8 from the control sites). The mean age of the patients was $32.24 \pm 11.63$, with no major differences in the two sites (mean age of $29.27 \pm 9.3$ in periumbilical lesions and $33.06 \pm 12.11$ in the control sites). Also there are no significant differences of age in males and females: $30.05 \pm 14.22$ in periumbilical lesions of men and $30.22 \pm 16.01$ on lesions of the control sites in men, and $28.9 \pm 7.74$ in women with periumbilical nevi and $33.74 \pm 11.04$ in women with nevi from the control areas. From the 121 nevi we studied 78 are intradermal (20 from the periumbilical area and 58 from the control sites), 26 compound ( 4 on the periumbilical area and 22 in the control areas), 9 junctional ( 2 in the periumbilical area and 7 in the control sites). From all the nevi we studied, 6 are dysplastic, all of them located in the control sites and of compound histologic type.

The total score obtained for the every lesion varies between 0 and 6 , with a mean value of the score of $0.97 \pm 1.4$. The mean value for the periumbilical nevi is $0.92 \pm 1.38$ and the mean value of the nevi from the control sites is $1,03 \pm 1,58$, thus the score of the periumbilical nevi is not significantly different than the score of the nevi located in the control sites. $(p=0.7733)$. For females with periumbilical lesions the mean value of the score is $0.85 \pm 1.42$ and men with the same kind of lesion have a mean score of 1.16 $\pm 1.32(\mathrm{p}=0.6271)$. The control sites nevi also present no major differences between sexes (mean value for women of $1.02 \pm 1.58$ and form men of $1.05 \pm 1.58$ ).

Considering the score of the periumbilical nevi compared to the nevi on the control sites, we encountered the following differences: asymmetry was present in $7,69 \%$ ( 2 of the 26 cases) of periumbilical lesions and $9.27 \%$ of control sites lesions (chi-square $=0.914, \quad p=0.3390$ ); the absence of demarcation of melanocytes at lateral margins of the lesion was present in $7.69 \%$ of the periumbilical lesions and $4.21 \%$ in the control sites (chi-square $=0.385, \mathrm{p}=0.5351$ ); the lentiginous proliferation of melanocytes for three consecutive papillary ridges is encountered in $11.53 \%$ of the periumbilical lesions and $10.52 \%$ of the control sites nevi (chi-square $=0.385, \mathrm{p}=0.5351$ ); the nested and discohesive pattern with bridging between rete ridges was encountered in $23.07 \%$ of periumbilical nevi and $16.84 \%$ of nevi in the control sites (chi-square $=0.079$, $\mathrm{p}=0.7793$ ); intraepidermal melanocytes singly or in groups, above the basal layer over the length of three rete ridges were found in $7.69 \%$ of the periumbilical nevi and $4.21 \%$ of the control sites nevi (chisquare $=2.622, p=0.1054)$; the involvement of the hair follicle by melanocyte proliferation was present in $3.84 \%$ of the periumbilical lesions and $8.42 \%$ of the nevi in other sites (chi-square $=1.507, p=0.2169$ ); the absence of maturation of deep dermal melanocytes wasn't identified in periumbilical and was encountered in nevi of the control sites in $3.15 \%$ of the cases (chi-square $=16.862, p<0.0001$ ); melanocyte atypia including one of the above mentioned criteria was found in $7.69 \% \%$ of the periumbilical nevi and $12.63 \%$ of the control sites nevi (chi-square $=0.154$, $\mathrm{p}=0.6949$ ); the fibroplasia of the papillary dermis was encountered in $11.53 \%$ of the periumbilical nevi and $16.84 \%$ of the control sites nevi (chi-square $=0.087$, $\mathrm{p}=0.7675$ ); lymphocytic infiltrate in the dermis was present with different intensities in $11,53 \%$ of the breast nevi and $16.84 \%$ of the control sites nevi (chisquare $=0.004, p=0.9478$ ).

We performed immunohistochemical examination on nevi with morphologic score higher than 3 ( $n=4$ for the periumbilical area and $n=14$ for the control sites). This revealed different degrees of positive reaction for the melanocytary markers in all the selected nevi regardless their location. We found a correlation between the gender of the patients and the expression intensity of Melan A and HMB45, this expression being higher in female patients. The proliferation marker Ki 67 had a negative expression in all the cases from the periumbilical area $(n=4)$ and for the control sites it had a negative expression in 6 cases (54.54\%), weak expression in 5 cases $(45.45 \%)$ and moderate expression in 3 cases $(27.27 \%)$. The Ki 67 expression correlates for the control sites with the morphologic score, therefore it correlates with the atypical changes. On the selected cases for immunohistochemical examination we practiced hormonal stains, particularly $\alpha$-ER. The reaction to $\alpha$-ER was not suggestive; all the cases had a negative expression regardless the location or the gender of the patient. 


\section{Discussions}

It is known for many years that a group of nevi in different anatomic sites that may simulate both dysplastic nevi and melanoma [1,6,7,12]. These nevi have been termed generically "nevi of special sites". As said above, the anatomical sites that have been included are acral locations, genitals, breast, scalp, ear, flexural regions and the conjunctiva. Some of these locations offer a physical explanation for the atypical features of the nevi (thick corneum and dermatoglyiphs of the soles and palms) but it is very speculative $[13,14]$. Most of the nevi from these anatomic sites are identical clinical and histological with the "banal" nevi (torso, arms except acral regions and other locations), and only a small part of them show atypical features that overlap dysplastic nevi or melanoma. Therefore the anatomical arguments, hormonal influences or effects of repeated friction are not sufficient to explain their atypical aspect. Even though they may show severe modifications their behavior is completely benign. The acral nevi tend to be more cellular than most of the nevi, with a predominant lentiginous than nested pattern and occasionally with pagetoid cells $[8,9,10,15]$. The nevi of the genitalia present with confluent melanocytic nests, with various sizes and shapes, and more discohesive melanocytes. There may be present a lentiginous growth as well as adnexal involvement. The presence of pagetoid cells is uncommon in genital nevi $[15,16]$. The flexural sites include several locations such as axillary, umbilical, inguinal, antecubital, popliteal fossae, pubic, scrotal, perineal and perianal and depending on each constitution the folds of the neck and abdomen $[6,17,18]$.

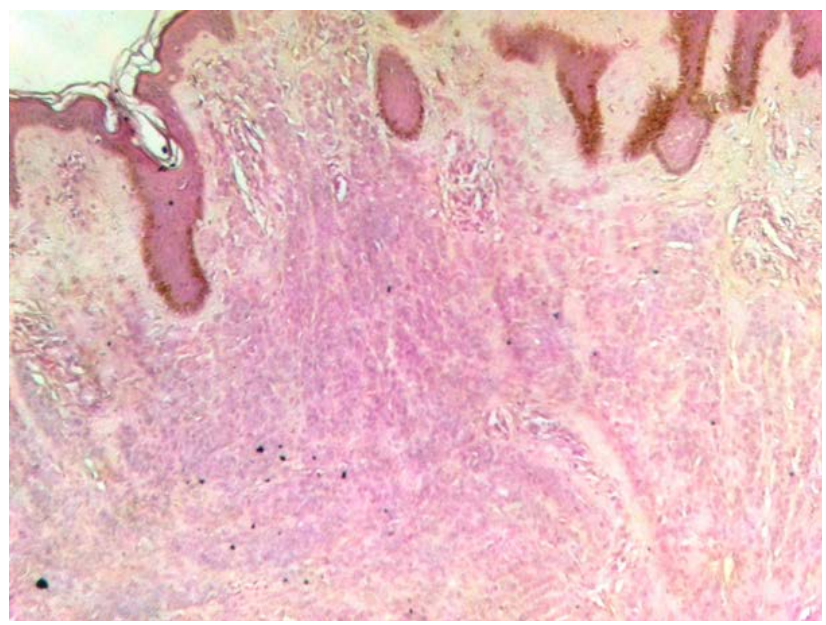

Figure 1 - Intradermal periumbilical nevus - confluence of the melanocytic nests, with diminished cohesion of melanocytes (HE x 20)

David E. Elder mentioned in an article published in 2006 in Modern Pathology that "it is just as important to recognize truly dysplastic nevi as such, as it is to avoid overdiagnosis of the nevi of special sites as dysplastic nevi, or as melanoma". In one study, Rongioletti et al. found $55.5 \%$ of the flexural nevi as atypical with features similar to genital nevi (pleomorphic nesting pattern and lack of cellular cohesion). Cytological atypia is not often found and when present it is mild and restricted to the junctional and papillary dermal component of the nevus. The morphology of the periumbilical nevi do not differ very much from the nevi in other locations, however they presented with more atypical features then nevi from other sites. The atypical features observed by Rongioletti et al. did dot correspond to any unusual biological behavior, meaning that they do not represent a risk for melanoma. Other studies (Hosler et al. 2008, J. Cutan Pathol - Nevi with siterelated atypia: a review of melanocytic nevi with atypical histolgic features based on anatomic site) revealed mmost of the times two atypical patterns for the flexural nevi, one the papilomatosis associated with rare cytological atypias and the other with melanocytic nests of various sizes and shapes, with cytological atypias at the dermo-epidermal junction, atypias that are more uniform than pleomorphic $[19,20,21]$.

We studied the periumbilical lesions as part of 
the milk line in order to observe if any connections are present due to their common embryologic origin. The results we obtained were not very different from the ones of Rongioletti et al., therefore the morphologic features the most present in our cases of periumbilical nevi were the architectural disorder of the nevus cells nests $(23,07 \%)$ (Figure 1), lentiginous proliferation (11,53\%) (Figure 2), dermal fibroplasias $(11,53 \%)$ and the dermal lymphocytic infiltrate $(11,53 \%)$ (Figure 3).

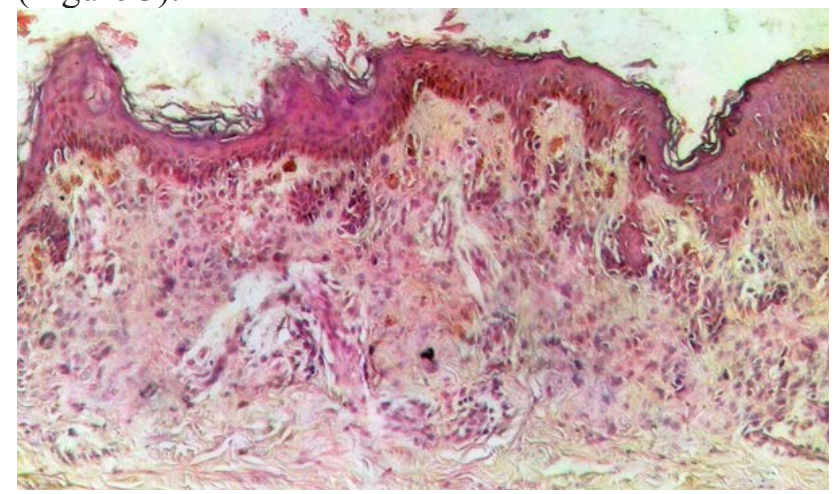

Figure 2 - Mixt periumbilical nevus - lentiginous proliferation of melanocytes over the length of more than three rete ridges (HE $x$ 10)

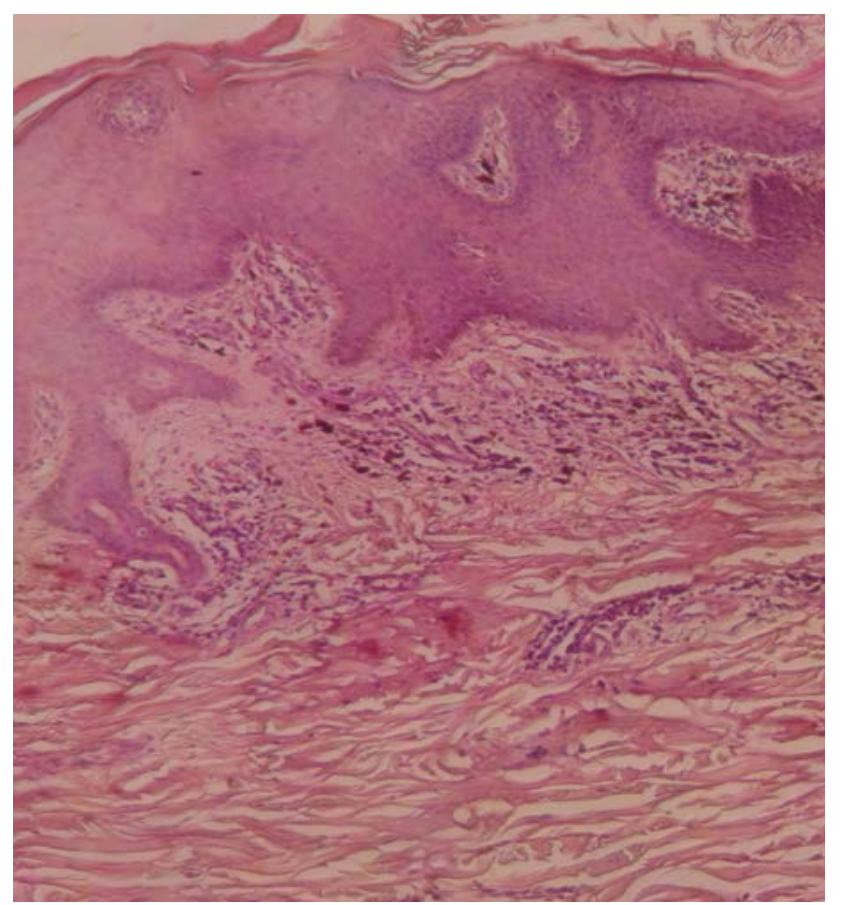

Figure 3 - Intradermal nevus - dermal lymphocytic infiltrate at the edges of the lesion (HE $\times 10)$
The difference between umbilical nevi and control sites nevi was not statistically significant, even if the atypical features tend to be more frequent for the periumbilical nevi.

There are no important differences between these values in men and women. Even though the expression of Melan A and HMB45 is higher in females, the limited number of cases that we performed immunohistochemical examination on does not offer a good statistical significance. Corroborating this aspect with the inconclusive immunohistochemical examination of estrogen receptors, we therefore considered that the modified aspect of the periumbilical nevi as part of the milk line is not hormonally influenced.

\section{Conclusions}

We can conclude that these atypical features that can be encountered in periumbilical nevi are only site related atypias so far not related to any hormonal influence. It is important for any pathologist to be aware of these changes on the periumbilical and other flexural sites nevi as well on other other specific sites (such as breast, ear, genital), to avoid overdiagnosis of malign melanoma and unnecessary concern from the patient.

\section{References}

1. Elder D.E. (2006). Precursors to melanoma and their mimics: nevi of special sites, Mod Pathol. 19 (Suppl 2), S4-S20.

2. Elder D.E., Elenitsas R., Johnson B., Murphy G.F. \& Xu G. (2009). Lever's histopathology of the skin, $10^{\text {th }}$ edition. (pp. 715-718). Philadelphia: Lippincott-Williams \& Wilkins 
3. Mason A.R., Mohr M.R., Koch L.H. \& Hood A.F. (2011). Nevi of special sites. Clin Lab Med. 31(2), 229-42.

4. Massi G. \& LeBoit P.E. (2004). Histological diagnosis of nevi and melanoma, $1^{\text {st }}$ edition. (pp. 329-331). Darmstadt: Philip E. Springer Steinkopff

5. Rongioletti F., Ball R.A., Marcus R. \& Barnhill R.L. (2000). Histopathological features of flexural melanocytic nevi: a study of 40 cases. $J$. Cutan Pathol. 27(5), 215-217.

6. Rongioletti F., Urso C., Batolo D., Chimenti S., Fanti P.A., Filotico R., Gianotti R., Innocenzi D., Lentini M., Tomasini C., Pippione M. \& Rebora A. (2004). Melanocytic nevi of the breast: a histologic case-control study, J Cutan Pathol. 31(2), 137-140.

7. Fabrizi G., Pagliarello C. \& Parente P. et al. (2007). Atypical nevi of the scalp in adolescents. J Cutan Pathol. 34, 365-369

8. Lazova R., Lester B. \& Glusac E.J. et al. (2005). The characteristic histopathological features of the nevi on and around the ear. J Cutan Pathol. 32, 42-44.

9. Saad A.G., Patel S. \& Mutasim D.F. (2005). Melanocytic nevi of the auricular region: histologic characteristic and diagnostic difficulties. Am. J. Dermatopathol. 27, 111-115

10. Kolm I., Kamarashev J., Kerl K., Moinetti C., Giovanoli P., French L.E. \& Braun R.P. (2011). Diagnostic pitfall: pigmented lesion of the nipple - correlation between dermoscopy, reflectance confocalmicroscopy and histopathoogy. Dermatology. 222(1), 1-4

11. Hosler G.A., Moresi J.M. \& Barrett T.L. (2008). Nevi with site-related atypia: a review of melanocytic nevi with atypical histologic features based on anatomic site. J Cutan Pathol. 35(10), 889-898

12. Elder D.E., Green M.H., Guerry D. $4^{\text {th }}$, Kraemer K.H. \& Clark W.H. Jr. (1982). The dysplastic nevus syndrome: our definition, Am. $J$. Dermatopathol. 4(5), 455-460

13. de Wit P.E., van't Hof-Grootenboer B., Ruiter D.J., Bondi R., Bröcker E.B., Cesarini J.P., Hastrup N., Hou-Jensen K., MacKie R.M. \& Scheffer E. et al. (1993). Validity of the histopathological criteria used for diagnosing dysplastic naevi. An inter-observer study by the pathology subgroup of the EORTC Malignant Melanoma Cooperative Group. Eur. J. Cancer. 29A(6), 831-839

14. MacKie R.M., English J., Aitchison T.C., Fitzsimons C.P. \& Wilson P. (1985). The number and distribution of benign pigmented moles (melanocytic naevi) in a healthy British population. Br J Dermatol. 113(2), 167-174

15. Boyd A.S. \& Rapini R.P. (1994). Acral melanocytic neoplasms: a histologic analysis of 158 lesions, J Am Acad Dermatol. 31(5Pt1), 740-745

16. Rongioletti F., Ball R.A., Marcus R. \& Barnhill R.L. (2000). Histopathological features of flexural melanocytic nevi: a study of 40 cases. $J$ Cutan Pathol. 27(5), 215-217

17. Fisher K.R., Maize J.C. Jr. \& Maize J.C. Sr. (2012). Histologic features of scalp melanocytic nevi, J Am Acad Dermatol._S0190-9622(12), 1182-6

18. Christensen W.N., Friedman K.F. \& Woodruff J.D. et al. (1987). Histologic characteristics of vulvar nevocellular nevi. J Cutan Pathol. 14, 8791

19. LeBoit P.E. (2000). A diagnosis for maniacs. $A m$ J Dermatopathol. 22(6), 556-558

20. Clark W.H. Jr., Hood A.F., Tucker M.A. \& Jampel R.M. (1998). Atypical melanocytic nevi of the genital type with a discussion of reciprocal parenchymal-stromal interactions in the biology of neoplasia, Hum Pathol. 29(1 Suppl 1), S1-S24

21. Blum A. \& Maltagliati-Holzner P. (2011). Monitoring a melanocytic tumor. When is excision indicated? Hautarzt._62(10), 774-777 